\title{
ARQUEOLOGÍA DE SUPERFICIE EN LA CUENCA DEL PAPALOAPAN, VERACRUZ.
}

\section{SURFACE ARCHEOLOGY IN THE PAPALOAPAN BASIN, VERACRUZ.}

\section{María Antonia Aguilar Pérez ${ }^{1}$}

antoaguilar@uv.mx

\section{RESUMEN}

A partir de la segunda mitad del siglo pasado la perspectiva respecto a la arqueología ha cambiado, se deja de percibir como una actividad de recopilación de objetos para dar paso verdaderas interpretaciones respecto a la vida y cultura pasada, para ello se requirió de cambios técnicos, metodológicos y por supuesto epistemológicos, es así que cobra importancia la arqueología de superficie. Con los estudios que se han realizado bajo estas perspectivas, en el desarrollo de investigaciones se observa ya como una práctica recurrente la arqueología de superficie y en el caso del Estado de Veracruz no es la excepción, la Cuenca del Papaloapan cuenta con exploraciones arqueológicas que permiten conocer parte del desarrollo socio cultural de la región.

Palabras clave: Arqueología de superficie, patrón de asentamiento, Cuenca del Papaloapan

\footnotetext{
${ }^{1}$ Facultad de Antropología, Universidad Veracruzana, México.
} 


\begin{abstract}
ABSTRAC
From the second half of the last century the perspective on archeology has changed, it is no longer perceived as an activity of collecting objects to make way for true interpretations regarding life and past culture, for this it required technical changes, methodological and of course epistemological, is how surface archeology becomes important. With the studies that have been carried out under these perspectives, in the development of investigations, surface archeology is already a recurrent practice and in the case of the State of Veracruz it is not the exception, the Papaloapan Basin has archaeological explorations that allow know part of the socio-cultural development of the region.
\end{abstract}

Keywords: Surface archeology, settlement pattern, Papaloapan Basin

\title{
CONTEXTO DE LA INVESTIGACIÓN
}

La arqueología desde sus inicios se ha identificado por los objetos recuperados de culturas antiguas alrededor del mundo, especialmente aquellos que conservan características de forma y decoración completa, pues son considerados como auténticos tesoros y se les atribuye un alto valor monetario, sin embargo, a lo largo de la historia y con el desarrollo de esta disciplina la perspectiva ha cambiado, especialmente para quienes se dedican profesionalmente a su estudio.

Desde los postulados de Darwin se empiezan a plantear nuevas consideraciones respecto a la visión del mundo, a partir de la segunda década del S. XX, las temáticas relacionadas con la geografía, la biología, y la antropología empiezan a cruzar caminos, a partir de los que se van a generar críticas para dar paso a nuevas propuestas con base en postulados epistemológicos; por ejemplo, Arnold y Osorio 
(1998) menciona los planteamientos de Bertalanffy a cerca de la Teoría General de Sistemas, la cual se define como:

“...como conjuntos de elementos que guardan estrechas relaciones entre sí, que mantienen al sistema directo o indirectamente unido de modo más o menos estable y cuyo comportamiento global persigue, normalmente, algún tipo de objetivo".

Si bien esta teoría fue muy criticada, no se pueden negar los aportes hechos a la arqueología, ya que de acuerdo con Patty Jo Whatson, Steven A. LeBlanc y Charles Redman (1974) intenta poner en relación conjuntos de datos, busca un orden posible para focalizar de manera más general cualquier objeto, entre otras cosas, y de donde se desprende que los arqueólogos y por ende la arqueología, no deben de estudiar artefactos o actividades sino relaciones internas dentro de un sistema así como descubrir la forma en la que el sistema actúa en un medio ambiente dado; con estos antecedentes surgen nuevas preguntas y estudiosos que intentan responder a ellas.

Es así como Julian Steward plantea la Ecología Cultural, el afirma que:

“...La ecología cultural difiere de la ecología humana y social porque busca explicar el origen de los rasgos culturales particulares y los patrones que caracterizan diferentes áreas, en lugar formular principios generales aplicables a cualquier situación cultural-ambiental. Se distingue de las concepciones relativistas y neo-evolucionistas de la historia cultural en la medida en que introduce el medio ambiente local Indexadores: Latindex, ISIS, Google Academic 
como un factor extracultural en la premisa infructuosa de que la cultura proviene de la cultura." (1955)

Steward define y plantea un método para el estudio de la cultura, se puede resumir que se refiere al estudio de la relación de medio ambiente con el asentamiento y la organización social, en relación a estos aspectos es su alumno Gordon Willey quien realiza una investigación respecto a patrones de asentamientos en el valle del Virú en Perú; donde los define como:

"La manera en que el hombre dispone de sí mismo en el paisaje o medio en el que vive. Se refiere a casas, a sus arreglos y a la naturaleza y disposición de otras estructuras pertenecientes a la vida comunitaria... pueden ofrecer un punto de partida estratégico para la interpretación funcional de las culturas arqueológicas". (Willey, 1953).

\section{Arqueología de superficie y patrón de asentamiento}

Con el planteamiento de la Ecología Cultural y los estudios de patrón de asentamiento es que se puede considerar el inicio de la arqueología de superficie, con los principios teóricos derivados de la Teoría General de Sistemas donde se dice que la arqueología no busca objetos, se empiezan a dejar de lado exclusivamente la excavaciones para dar paso a investigaciones con un enfoque multidisciplinario, a partir de ese momento se reorientan los estudios hacia el comercio, las fronteras y las esferas de interacción de los sitios arqueológicos. 
Yoko Sigiura (2005) plantea que:

"El reconocimiento superficial a nivel macro y el análisis distribucional de los registros arqueológicos recuperados a nivel prospectivo nos permiten profundizar en diferentes aspectos del pasado, para definir, por ejemplo, en dimensiones espacio-temporales, el área ocupada por una cultura o por grupos étnicos, y así dilucidar el porqué o el cómo estaban organizadas las poblaciones de una región, y las relaciones coetáneas entre los asentamientos y las de éstos con su entorno ambiental".

Con base en los estudios de R. J. Ruppé, ella plantea que el desarrollo del reconocimiento de superficie se retrasó debido a la idea equivocada de que sólo podía ser utilizado como una etapa previa a la excavación, sólo para localizar y determinar el lugar a excavar.

En México los primeros trabajos con esta visión se desarrollan en Teotihuacán, Natalia Moragas (2003) nos indica que a inicio de los años 60's el Dr. Rene Millon comienza con un proyecto de recorrido de superficie para estudiar el área urbana del sitio: el Teotihuacan Mapping Project, donde se apoyó en la fotografía aérea y en excavaciones de pozos de sondeo, de este proyecto se origina el mapa del sitio con tal detalle que aún se continúa utilizando.

La misma autora nos indica de otro proyecto que emplea el recorrido de superficie como base de investigación, el del Dr. William Sanders: Teotihuacan Valley 
Project, donde se busca determinar la densidad de la población en el valle, en este caso el objetivo es el área rural, “...Se localizaron cerca de 600 sitios arqueológicos de los cuales se excavaron cerca de una veintena con la finalidad de resolver algunos de los problemas específicos surgidos en la prospección del territorio." (Moragas, 2003). De acuerdo con ella tanto el proyecto de área urbana (Millon) como el área rural (Sanders) determinaron que Teotihuacán, en su época, fue el centro urbano de mayor magnitud durante el periodo clásico en la Cuenca de México y de Mesoamérica. A partir de entonces los estudios de reconocimiento de superficie en México se desarrollaron con más frecuencia.

\section{Delimitación de áreas de estudio}

Un aspecto importante en la arqueología de superficie es el tipo de terreno donde se desarrollan las investigaciones, de ello dependerá en muchas ocasiones las estrategias a seguir para lograr alcanzar los objetivos, por lo cual debe de realizarse una delimitación de área misma que debe de valorarse desde el momento que se diseña la investigación; García San Juan (2005) explica que generalmente se aplican tres criterios para llevar a cabo la delimitación de un área de estudio; a) delimitación administrativa, b) delimitación poligonal ad hoc y c) delimitación geográfica y cultural. La primera obedece a criterios administrativos actuales de los terrenos como pueden ser parcelas catastrales, municipios, estados; el segundo criterio se vincula a intervenciones arqueológicas de emergencia, como 
puede ser el trazo de carreteras u obras de infraestructura y el tercer caso se refiere a unidades fisiográficas como puede ser un valle o una llanura.

\section{Cuenca del Papaloapan}

Ya para la última década del siglo XX el desarrollo de la arqueología de superficie es pleno, y la mayoría de las investigaciones son planteadas a partir de un diseño de investigación con planteamientos claros, en el estado de Veracruz se han desarrollado múltiples trabajos con este enfoque principalmente en áreas que tradicionalmente han sido investigadas, como la región olmeca, el centro y norte del estado; sin embargo también se empiezan a realizar trabajo en otras regiones poco atendidas, como es el caso de la Cuenca del Papaloapan.

La Cuenca del Papaloapan es una región muy importante pues se tiene registros históricos de poblaciones establecidas en la región al momento de la llegada de los españoles, y en documentos prehispánicos existen registros de asentamientos importantes que tributaban al grupo dominante en el periodo anterior al arribo de los ibéricos. Fray Toribio Motolinía en su "Historia de los indios de la Nueva España", da referencia de la zona del Papaloapan como lugar lleno de riquezas, donde los españoles obtuvieron tributos (aunque no especifica cuales fueron) y la repartieron entre ellos, pero fue tal su explotación que “...tanto la chuparon que la dejaron más pobre que otra..." (Motolinia.1995:163); sin embargo es hasta la 
última década del siglo $\mathrm{XX}$ que se empiezan a registrar investigaciones directamente allí.

La Cuenca del Papaloapan (fig. 1) es una región hidrográfica que se localiza en el sur del estado de Veracruz entre las cuencas de los ríos Jamapa y Coatzacoalcos, con una superficie estimada de $46500 \mathrm{Km} 2$. La mayor parte del territorio de la Cuenca son planicies o laderas con poca pendiente.

El geógrafo Joaquín González Martínez (2004), de acuerdo a los trabajos realizados para la Comisión del Papaloapan por Jorge Tamayo, dice que la Cuenca se divide fisiográficamente en dos áreas; el Alto y Bajo Papaloapan. En la primera se localizan las fuentes del complejo hidrológico en 127 municipios en el Estado de Oaxaca, 25 en Puebla y 35 en Veracruz; mientras la segunda área abarca desde los pie de monte de las sierras de Oaxaca, el eje Volcánico Transversal y el Macizo de Los Tuxtlas, hasta su desembocadura en la costa del Golfo de México; ubicándose en 23 municipios del Estado de Veracruz y 15 del Estado de Oaxaca. 


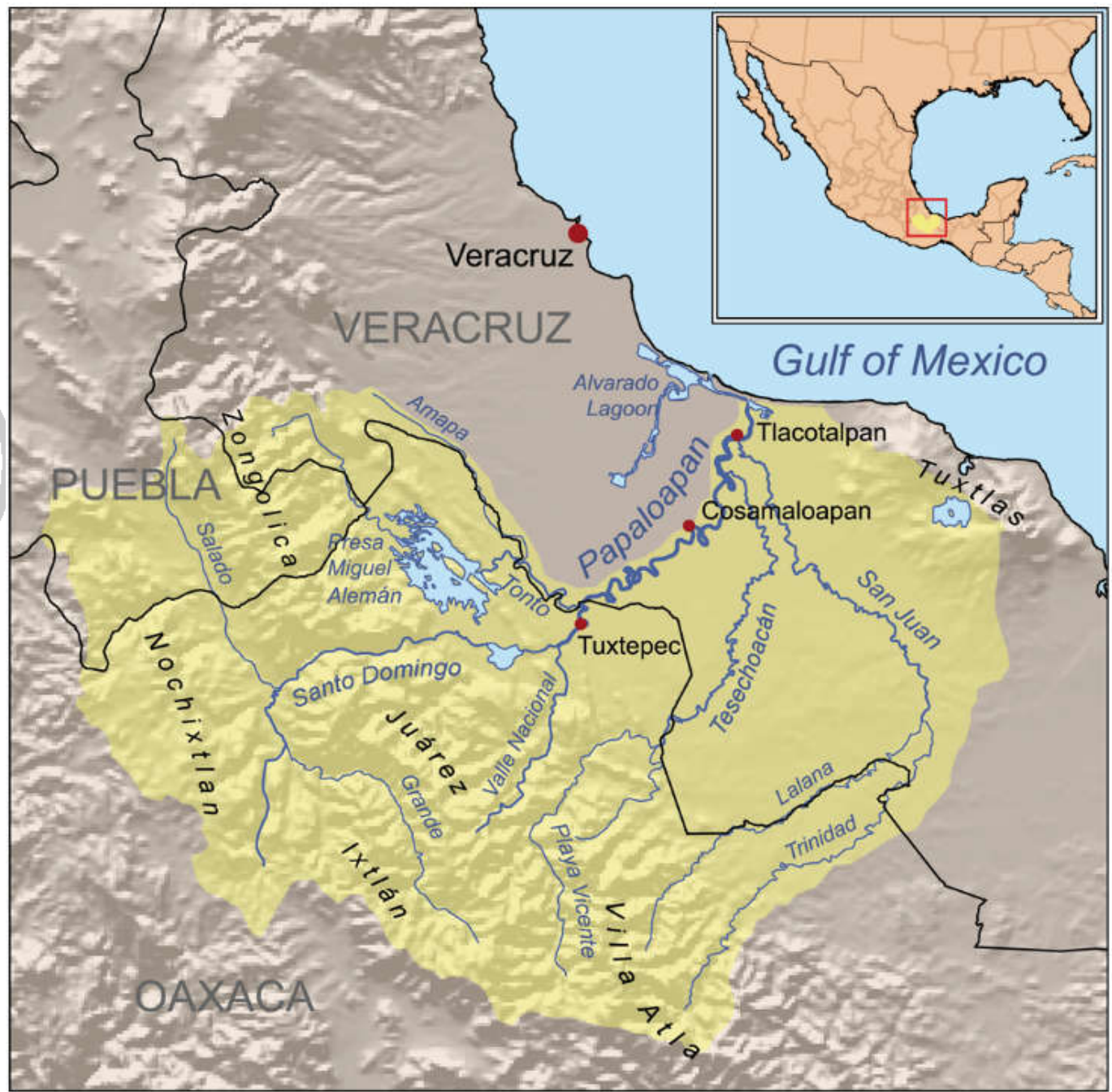

Fig. 1 Cuenca del Papaloapan, tomado de:

https://upload.wikimedia.org/wikipedia/commons/6/6c/Papaloapanrivermap.png 
El contraste que existe entre las dos secciones que conforman el Papaloapan es evidente. La parte alta es montañosa, “...lo que ha coadyuvado no sólo a mantener los altos valores hídricos..., sino a la formación de cañadas y valles...” (González Martínez. 2004:63). Por otro lado, en la parte baja comprende la llanura del Sotavento; con alturas máximas de 100m SNM aproximadamente en las zonas de pie de monte.

En los siguientes apartados se reseña los trabajos en los cuales la autora ha participado y que fueron realizados en la región de la Cuenca del Papaloapan, específicamente en los municipios de Tlacojalpan y Playa Vicente, ambos en el estado de Veracruz. (Fig. 2). 


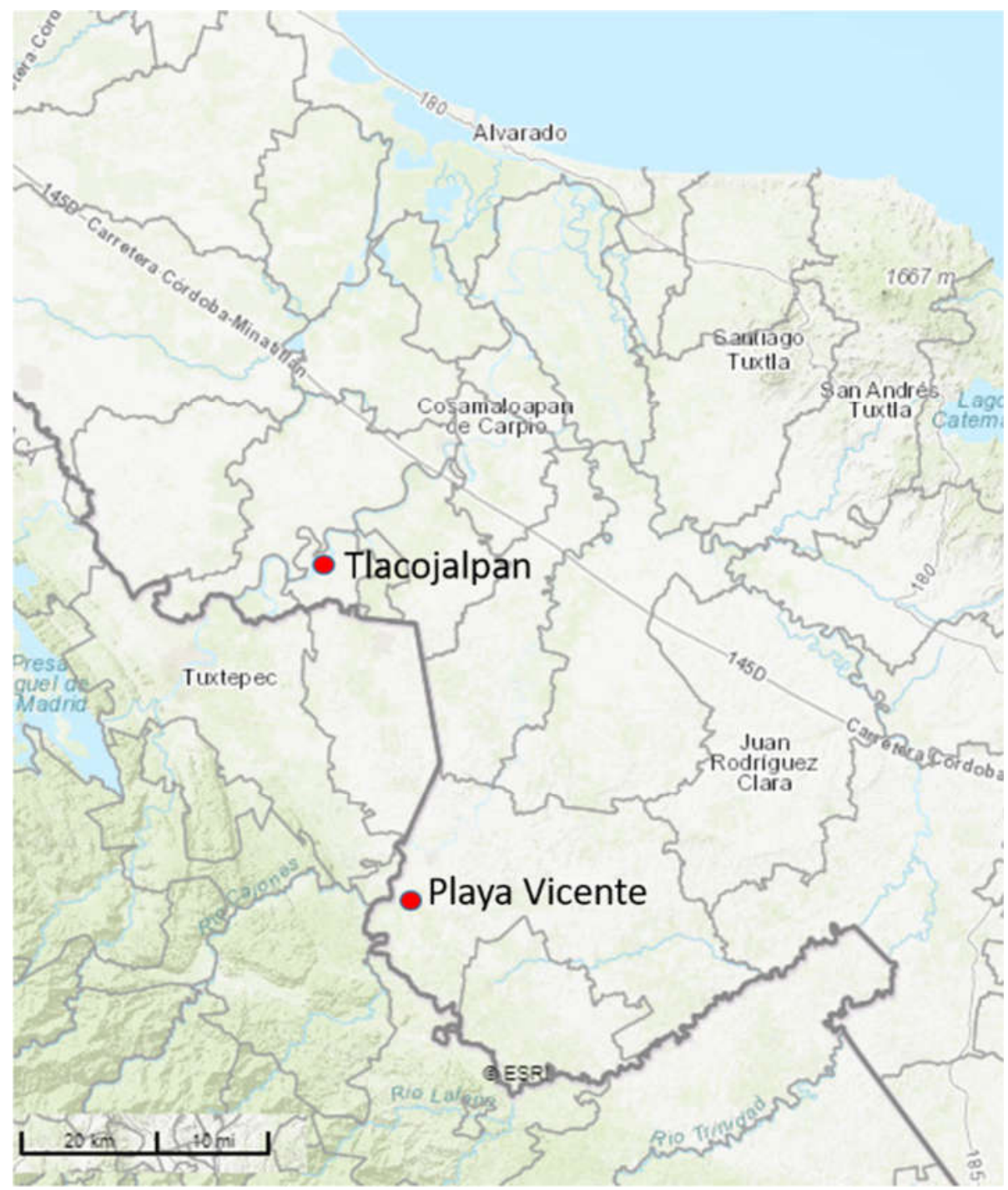

Fig. 2. Localización de los municipios de Tlacojalpan y Playa Vicente. 


\section{Proyecto Patrón de Asentamiento y Poblamiento en la Cuenca Baja del}

\section{Papaloapan.}

En 1998 por primera vez se inicia la realización de un estudio sistemático con el proyecto: Patrón de Asentamiento y Poblamiento Prehispánico en la Cuenca Baja del Papaloapan (PAYPPCUBAPA), dirigido por el Dr. Pedro Jiménez Lara, Investigador del Instituto de Investigaciones Histórico Sociales de la Universidad Veracruzana (2000).

El proyecto dio inicio como parte del programa interdisciplinario de estudios regionales, el cual tiene como objetivo conocer la historia de la región de la Cuenca Baja del Papaloapan, así como su estructuración y sus diversos periodos de desarrollo.

El proyecto, dirigido por el Dr. Jiménez, pretende un reconocimiento sistemático de la Cuenca Baja del Papaloapan, lo que se ha desarrollado trabajando por municipios, empezando en Otatitlán que es el municipio tomado como límite suroeste de la región planteada y continuando con Tlacojalpan.

La primera temporada de trabajos de campo se realizó en los municipios antes mencionados durante el año de 1999; el objetivo fue hacer un reconocimiento para detectar vestigios arqueológicos, así como elaborar sus respectivos levantamientos 
topográficos, recolección de materiales de superficie y pozos estratigráficos para, con su análisis, establecer una tipología y dar una idea de la cronología de área.

Durante la segunda temporada, se realizaron las colecciones de materiales de aquellas zonas donde faltaron de realizarse en la primera temporada, en este caso las unidades de los sitios Playa María y La Campana, así como la delimitación y levantamiento topográfico del sitio Soledad-San Marcos y sus respectivas colecciones de material de superficie. Hasta este momento los trabajos fueron realizados por el Dr. Jiménez y un equipo de trabajadores de las localidades estudiadas, con apoyo de un ingeniero topógrafo y una arqueóloga, en adelante el Dr. Jiménez incorpora estudiantes de la Universidad Veracruzana de la Licenciatura en arqueología, con lo cual el proyecto contribuye en la formación de nuevas generaciones de arqueólogos. (Fig. 3)

Para el año 2001, se llevó a cabo la tercera temporada, durante la cual se realizó una excavación en el sitio La Campana, y se continuó con el recorrido de superficie, donde se detectaron una serie de campos elevados ubicados al norte del actual poblado de Tlacojalpan. Las siguientes temporadas de trabajo de campo se continuaron localizando sitios arqueológicos, entre ellos un sitio de características particulares; se trata del sitio identificado como El Socorro, de acuerdo con Ruíz Cabañas (2008) se trata de un asentamiento: 
“...formado por dos líneas de montículos, una frente a la otra y que presenta la evidencia de una depresión longitudinal en medio de ella, que presumiblemente es a consecuencia de la extracción de la tierra para la creación de dichos montículos..., el sitio es seccionado de manera transversal por una laguna"

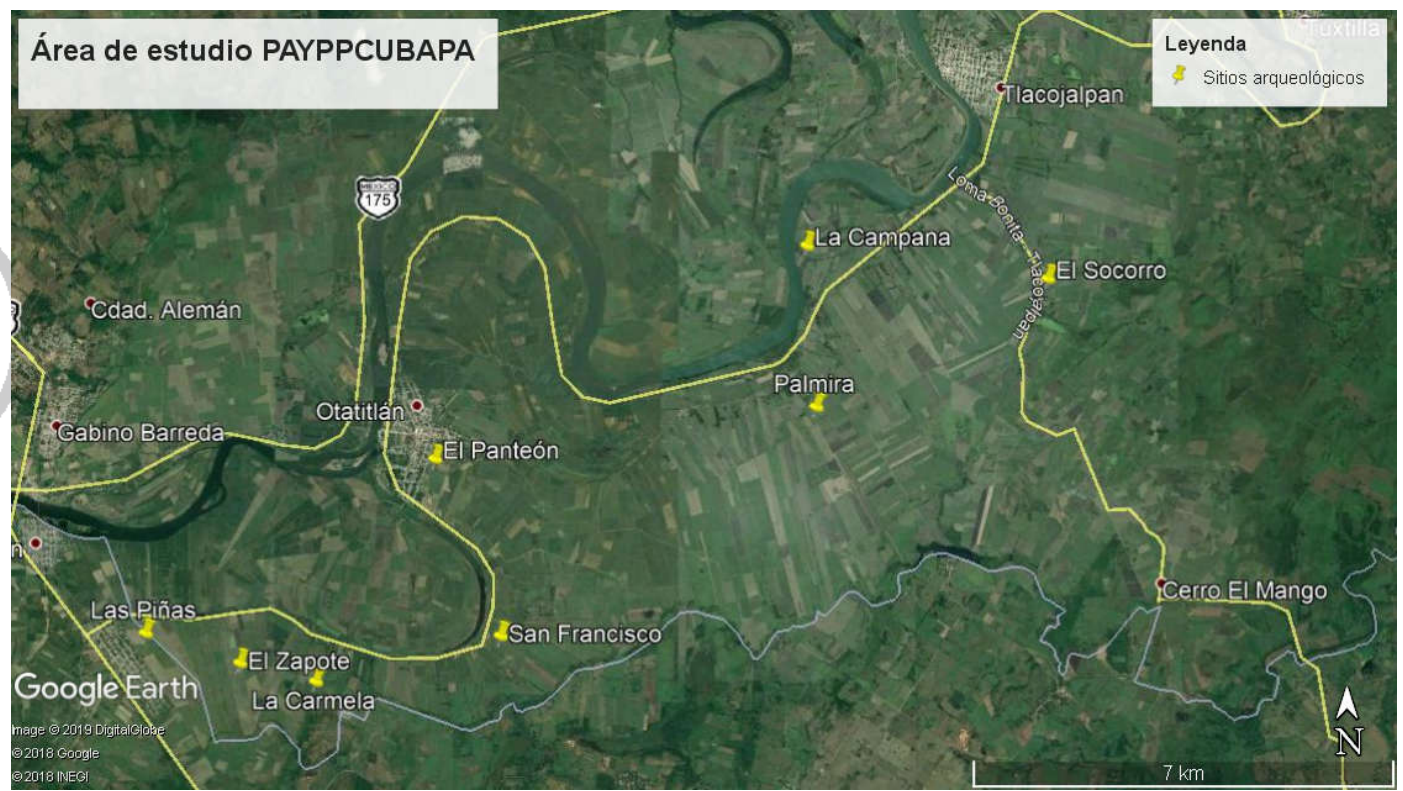

Fig. 3. Localización de sitios en el área de estudio del PAYPPCUBAPA.

\section{Proyecto Reconocimiento Arqueológico en la Región de Playa Vicente, Veracruz}

En 1997, quién esto suscribe llevó a cabo un trabajo recepcional donde se hace el análisis de los antecedentes de la región, sin embargo, se logra ampliar parcialmente la información en materia arqueológica, pues se ubican veintiocho 
rasgos, entre sitios y montículos aislados, esto por medio de la fotografía aérea y una inspección sobre terreno que se realizó para verificar las marcas localizadas durante la fotointerpretación, el trabajo no incluyó recolección alguna de material ni levantamientos topográficos, puesto que el objetivo fue plantear una propuesta para la protección de sitios arqueológicos, a través de la delimitación de zonas de posible impacto de crecimiento poblacional. (Aguilar.1997).

A partir del año 2005 da inicio el proyecto "Reconocimiento Arqueológico en la Región de "Playa Vicente Ver.", dirigido por quien suscribe este documento; allí se plantea un reconocimiento que busca el registro y delimitación de sitios arqueológicos en el área propuesta, para con ello contribuir al conocimiento de la historia regional y divulgación para la protección del patrimonio histórico cultural del área por medio del registro preciso de los restos arqueológicos existentes en la región. (Fig. 4). 

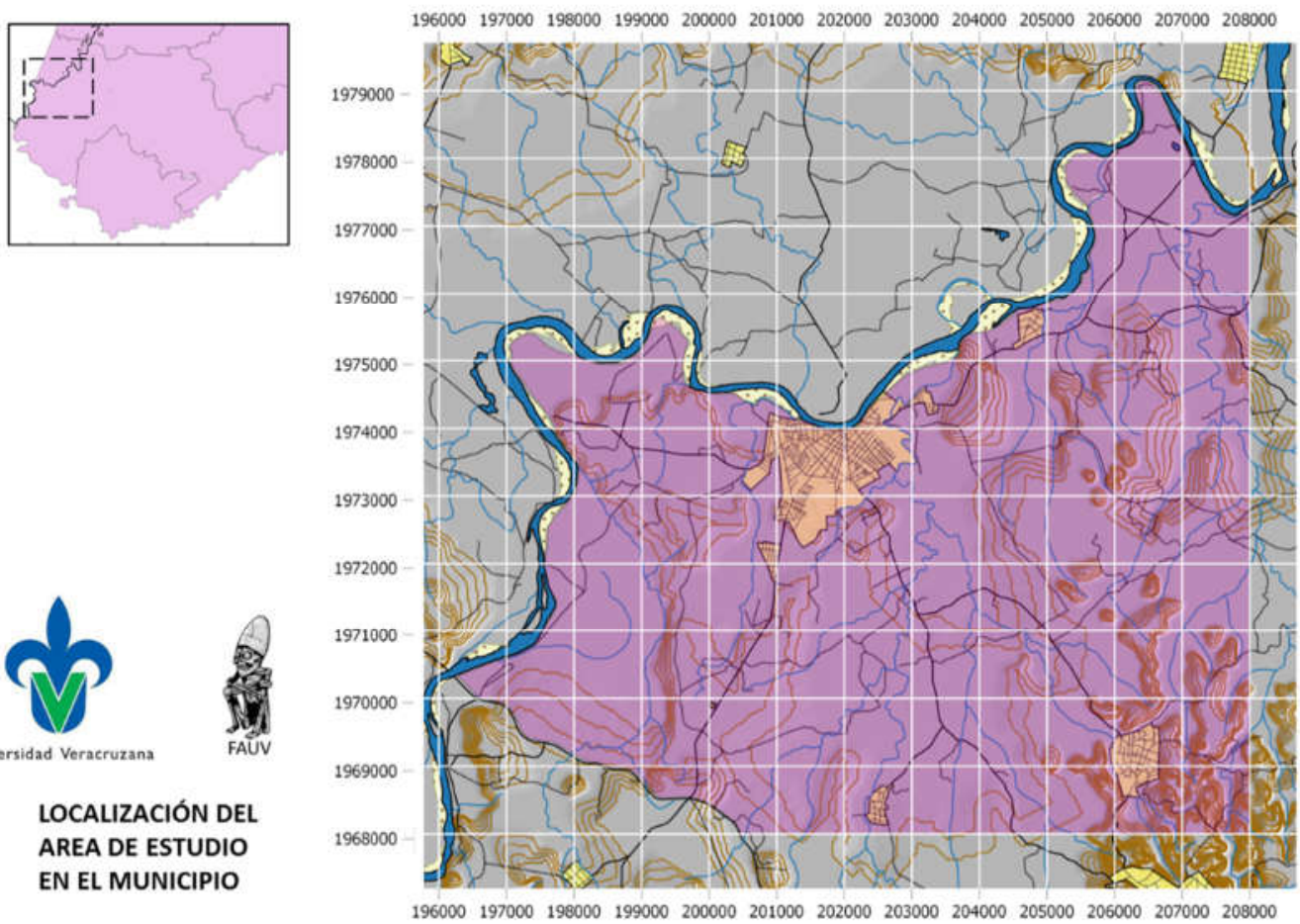

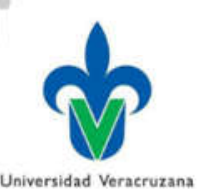

LOCALIZACIÓN DEL AREA DE ESTUDIO EN EL MUNICIPIO

Fig. 4. Área de estudio, Proyecto Playa Vicente.

En un artículo publicado en 2017, se exponen los avances que se han logrado en el proyecto (Aguilar 2017), allí se describen las características de los sitios localizados, así como las posibles cronologías.

En el área de estudio todos los montículos registrados tienen un sistema constructivo a base de tierra apisonada, su distribución es variada, podemos 
encontrar conjuntos bien definidos como pequeñas plazas, con montículos cónicos en los extremos y otros alargados hacia los costados, cuyas alturas oscilan desde los cincuenta centímetros a los tres metros para las estructuras alargadas y de uno a 13 metros para las cónicas. También se tienen concentraciones de montículos pero que no se distribuyen con un patrón específico, sin embargo, alcanzan elevaciones hasta de quince metros a partir de su base, o incluso algunos de ellos aislados cuya altura también varía entre uno y cinco metros, a cualquiera de estas construcciones se les ha llamado conjunto arquitectónico. También se identifican elevaciones con ocupación, que son lomas naturales aprovechadas las cuales pueden tener o no una modificación para establecer en ellas unidades habitacionales o alguna actividad antrópica.

\section{Estrategias de trabajo}

Para el caso de las áreas arriba expuestas es pertinente mencionar que la delimitación de las mismas se decidió con base en la combinación de criterios, por un lado se tomaron en cuenta rasgos geográficos como los ríos, para el caso de Tlacojalpan en rio Papaloapan, y en Playa Vicente el rio Tesechoacán; el otro criterio empleado fue administrativa en el primer municipio, pues el límite es precisamente la delimitación territorial del municipio, y para el segundo municipio se tomó una coordenada UTM que coincide aproximadamente con un cambio de elevación en el terreno. 
En ambos casos se utilizó la técnica de transectos para realizar los recorridos, esta consiste en establecer áreas más pequeñas para ir cubriendo terreno en líneas rectas separadas entre sí a determinada distancia, una persona puede caminar por la línea que se orienta con apoyo de una brújula o GPS y debe de observar al terreno a ambos lado de ella para registrar los vestigios que pudieran estar presentes, para una cobertura adecuada en la región de estudio la separación entre cada transecto generalmente se dio a veinte metros, y el área era determinada por los límites de terrenos o cercas, de modo que se pudiera obtener una cobertura total.

Un aspecto importante que debe de mencionarse es el uso de suelo, ya que de ello dependió la observación y registro de los rasgos arqueológicos, ya sean arquitectónicos o concentración de materiales.

En Tlacojalpan la mayor parte de los terrenos están dedicados a la agricultura de caña de azúcar y en menor medida al cultivo de banano y mango, lo cual generó algunas dificultades para el reconocimiento de superficie, pues en aquellas áreas donde la caña estaba desarrollada a más de cincuenta centímetros de altura era mayor el problema para identificar elevaciones del terreno que pudieran indicar rasgos arqueológicos, además para la siembra y producción se necesita de una penetración de cuchillas metálicas de alrededor de treinta centímetros o más, lo cual va deteriorando los restos arqueológicos, sobre todo porque al ser una región 
de planicie aluvial los elementos arquitectónicos fueron elaborados a base de tierra. Sin embargo, al tener la oportunidad de realizar varias temporadas de trabajo de campo se pudo cubrir la mayoría del área.

En Playa Vicente, como ya se mencionó, también se utilizó la técnica de cobertura total por medio de transectos, sin embargo los problemas que se enfrentaron fueron un tanto distintos por el tipo de uso de suelo, en este municipio la actividad principal es la ganadería, por lo cual se requiere de pastizales, en este caso el inconveniente fue el pasto pues si bien se pueden observar los rasgos arquitectónicos, que para esta área se han conservado en gran medida gracias a la misma actividad económica, lo que es muy difícil de observar y registrar son elementos como cerámica o lítica, para obtener muestras se debió de poner mucha atención en áreas donde el pasto fuera escaso, en las orillas de las cercas, o donde la fauna local tuviera algún impacto como para mostrar algunos ejemplares de material arqueológico.

En ambos casos las colecciones de muestras de materiales arqueológicos tienen la característica de ser muy erosionadas y fragmentadas, lo cual dificultó la identificación y comparación con materiales de otras áreas con la finalidad de establecer comparaciones, con las cuales se pudieran dar propuestas respecto a las respuestas de las preguntas que se plantearon originalmente; razón por la cual se debió de recurrir excavaciones de sondeo y con los resultados de ellas proponer 
por ejemplo una cronología y la distinción entre los diferentes asentamientos tanto en tiempo como en espacio.

A pesar de la dificultad para establecer una cronología, la ubicación de los sitios gracias a la arqueología de superficie, ha enriquecido el conocimiento de la región pues ahora se cuenta con registros sistemáticos de sitios arqueológicos, algunos de ellos con estructuras arquitectónicas bien definidas en conjuntos o agrupaciones, en otros casos montículos aislados o pequeñas concentraciones de material cerámico o lítico y con el apoyo de la excavación se complementan los datos para seguir generando interpretaciones y planteando nueva interrogantes.

\section{REFERÉNCIAS BIBLIOGRÁFICAS}

AGUILAR Pérez, María Antonia, Propuesta para la conservación del Patrimonio cultural: Playa Vicente, Ver, Tesina de licenciatura. Xalapa, Ver., México. Universidad Veracruzana, 1997.

La Playa de Vicente. Cronología de sitios prehispánicos en la región del Río Tesechoacán. En: Arqueología de la Costa del Golfo. Dinámicas de la interacción política e ideológica. En L. Budar, M. 1. Venter y S. Ladrón de Guevara. Xalapa, Ver. Universidad Veracruzana, 2017.

ARNOLD, Marcelo, Osorio, Francisco, Introducción a los Conceptos Básicos de la Teoría General de Sistemas. Cinta de Moebio [en línea], [Fecha de consulta: 1 de mayo de 2019] Disponible en:<http://www.redalyc.org/articulo.oa?id=10100306> ISSN.

GARCÍA San Juan, Leonardo, Introducción al reconocimiento y análisis arqueológico del territorio. Barcelona. Ariel, 2005. 
GONZÁLEZ Martínez, Joaquín Roberto, Historia social de Alvarado y su región. Xalapa, Ver. Universidad Veracruzana, 1998.

JIMÉNEZ Lara, Pedro, Proyecto "Patrón de Asentamiento y Poblamiento Prehispánico en la Cuenca Baja del Papaloapan, Ver. Xalapa, Ver. Universidad Veracruzana/CONACYT, 1998.

MORAGAS, Natalia, Dinámica del cambio cultural durante el epiclasico, 650-900 Teotihuacan México. Tesis de doctorado. Universitat de Barcelona, 2003.

MOTOLINÍA, Fray Toribio, Historia de los indios de la Nueva España. Estudio crítico, apéndices, notas e índice de Edmudo O’Gorman. México. Edit. Porrua. Col. Sepan Cuantos, num. 129, 1995.

RUÍZ Cabañas, Ángel, El Socorro un complejo residencial prehispánico en el Bajo Papaloapan, municipio de Tlacojalpan, Ver. Tesis de licenciatura. Xalapa, Ver. Facultad de Antropología. Universidad Veracruzana, 2008.

Steward Julian H. "El concepto y el método de la Ecología Cultural”, en: Clásicos y Contemporáneos en Antropología, CIESAS-UAM-UIA Cap. 2, de Theory of Culture Changes, University of Illinois Press, Urbana, 1955. [Fecha de consulta: 1 de mayo de 2019] Disponible: en:

http://www.ciesas.edu.mx/publicaciones/clasicos/00_CCA/Articulos_CCA/CCA_PDF/04 0_STEWARD_1955_El\%20Concepto_yel_metodo.pdf

SIGIURA, Yoko,Y detrás quedó la ciudad de los dioses. Historia de los asentamientos en el valle de Toluca, México, IIA-UNAM, 2005.

WHATSON, Patty Jo; STEVEN A. LeBlanc; REDMAN, Charles, El método científico en arqueología. Madrid. Edit. Alianza, 1974.

WILLEY, Gordon, Prehistoric Settlement Patterns in the Viru Valley, Peru. Bulletin 155 Smithsonian Institution, Bureau of American Ethnology, Washington, D.C, 1953. 